\title{
ON THE DISTANCE OF THE RIEMANN-LIOUVILLE OPERATOR FROM COMPACT OPERATORS
}

\author{
BOHUMIR OPIC
}

(Communicated by Palle E. T. Jorgensen)

ABSTRACT. We consider generalized Hardy operators

$$
T f(x)=\int_{a}^{x} \varphi(x, y) f(y) d y, \quad x \in(a, b) \subset \mathbb{R},
$$

acting between two weighted Lebesgue spaces $X=L^{p}(a, b ; v)$ and $Y=$ $L^{q}(a, b ; w), 1<p \leq q<\infty$, and present lower and upper bounds on the distance of $T$ from the space of all compact linear operators $P, P: X \rightarrow Y$. The conditions on the kernel $\varphi(x, y)$ are patterned in such a way that the above mentioned class of operators $T$ contains the Riemann-Liouville fractional operators of orders equal to or greater than one.

\section{INTRODUCTION}

Let $-\infty \leq a<b \leq \infty$. By $\mathscr{W}(a, b)$ we denote the set of all weights, i.e., the set of all functions measurable, positive, and finite almost everywhere on $(a, b)$. For $p \in(1, \infty)$ and $v \in \mathscr{W}(a, b)$ the weighted Lebesgue space $L^{p}(a, b ; v)$ is defined as the set of all measurable functions on $(a, b)$ with a finite norm

$$
\|u\|_{p,(a, b), v}=\left(\int_{a}^{b}|u(x)|^{p} v(x) d x\right)^{1 / p} .
$$

(If $v \equiv 1$, we write simply $L^{p}(a, b)$ and $\|\cdot\|_{p,(a, b)}$ instead of $L^{p}(a, b ; v)$ and $\|\cdot\|_{p,(a, b), v}$, respectively.)

Let $1<p \leq q<\infty, v, w \in \mathscr{W}(a, b)$. Several authors (cf. [6, 8, 2]) have established necessary and sufficient conditions for the inequality

$$
\|T f\|_{q,(a, b), w} \leq C\|f\|_{p,(a, b), v}
$$

to hold on $L^{p}(a, b ; v)$, where the operator $T$ is given by

$$
T f(x)=\int_{a}^{x} \varphi(x, y) f(y) d y
$$

with a kernel $\varphi$ satisfying various assumptions. Here we consider the kernel $\varphi$

Received by the editors January 12, 1993.

1991 Mathematics Subject Classification. Primary 47B38, 26A33; Secondary 26D10. 
with the following properties (cf. [2]):

(i) $\varphi:\left\{(x, y) \in \mathbb{R}^{2}: x, y \in(a, b), x>y\right\} \rightarrow(0, \infty)$;

(ii) $\varphi(x, y)$ is nondecreasing in $x$ and nonincreasing in $y$;

(iii) there exists $D>0$ such that

$$
\varphi(x, y) \leq D[\varphi(x, z)+\varphi(z, y)] \text { if } y<z<x .
$$

The Riemann-Liouville operator $T_{\sigma}$, given by

$$
T_{\sigma} f(x)=\int_{0}^{x}(x-y)^{\sigma-1} f(y) d y, \quad x>0, \sigma \geq 1,
$$

is an example of an operator $T$ from (1.2) whose kernel satisfies (1.3)(i)(1.3)(iii). Note that in all papers mentioned above it is assumed that $a=0$, $b=\infty$, and the kernel $\varphi$ is such that conditions (1.3)(i)-(1.3)(iii) are fulfilled. Set

Let $p^{\prime}=p /(p-1), q^{\prime}=q /(q-1), a \leq \alpha<\beta \leq b, t \in(\alpha, \beta), i \in\{0,1\}$.

$$
\begin{gathered}
B_{(\alpha, \beta)}^{(i)}(t)=\left(\int_{t}^{\beta} \varphi(x, t)^{q i} w(x) d x\right)^{1 / q}\left(\int_{\alpha}^{t} \varphi(t, x)^{p^{\prime}(1-i)} v(x)^{1-p^{\prime}} d x\right)^{1 / p^{\prime}}, \\
B_{(\alpha, \beta)}^{(i)}=\sup _{\alpha<t<\beta} B_{(\alpha, \beta)}^{(i)}(t), \quad B_{(\alpha, \beta)}=\max _{i=0,1} B_{(\alpha, \beta)}^{(i)} .
\end{gathered}
$$

Applying the method of [6] one can prove

Theorem 1.1. Let $1<p \leq q<\infty$, and let the function $\varphi$ satisfy (1.3)(i)(1.3)(iii). Then there exists a positive constant $C$ such that inequality (1.1) holds for all $f \in L^{p}(a, b ; v)$ if, and only if,

$$
B_{(a, b)}<\infty .
$$

Moreover, if $C$ is the least constant for which (1.1) holds on $L^{p}(a, b ; v)$, then

$$
B_{(a, b)} \leq C \leq k(q, p, D) B_{(a, b)},
$$

where

$$
k(q, p, D)=2^{1 / q^{\prime}}(D+1)^{2}\left[D^{q}\left(1+\frac{q}{p^{\prime}}\right)\left(1+\frac{p^{\prime}}{q}\right)^{q / p^{\prime}}+1\right]^{1 / q}
$$

with $D$ from (1.3)(iii).

In other words, Theorem 1.1 provides a necessary and sufficient condition for the boundedness of $T$ from $X:=L^{p}(a, b ; v)$ into $Y:=L^{q}(a, b ; w)$, $1<p \leq q<\infty$. The aim of the paper is to establish lower and upper bounds on the distance of $T$ from the space of all compact linear operators from $X$ into $Y$ provided $T: X \rightarrow Y$ is bounded and the function $\varphi$, in addition to conditions (1.3)(i)-(1.3)(iii), satisfies

(1.3)(iv) For any $\gamma \in(a, b)$ the function $\varphi$ is uniformly continuous on the set $\left\{(x, y) \in \mathbb{R}^{2}: x, y \in(a, b), y<x<y\right\}$.

(Note that for the Riemann-Liouville operator $T_{\sigma}$ from (1.4) condition (1.3)(iv) is again fulfilled.) In particular, from such bounds one easily obtains 
a characterization of those operators $T$ that are compact. The main result of the paper is Theorem 3.1. Our work is patterned on $[10,5,4]$, where operators $P: L^{p}(a, b) \rightarrow L^{q}(a, b)$, given by

$$
P f(x)=v(x) \int_{a}^{x} v(y) f(y) d y, \quad x \in(a, b),
$$

with $v, w \in \mathscr{W}(a, b)$, are considered. We also use some techniques drawn from $[9,3]$.

\section{Preliminaries}

Let $X, Y$ be Banach spaces. The symbol $\mathscr{B}(X, Y)$ is used to denote the space of all bounded linear maps from $X$ into $Y$. The subset of $\mathscr{B}(X, Y)$ consisting of all compact maps or maps having a finite-dimensional range is denoted by $\mathscr{K}(X, Y)$ or $\mathscr{F}_{r}(X, Y)$, respectively. Evidently, for any $P \in \mathscr{B}(X, Y)$,

$$
\operatorname{dist}(P, \mathscr{K}(X, Y)) \leq \operatorname{dist}\left(P, \mathscr{F}_{r}(X, Y)\right) \text {. }
$$

It is easy to see that the map

$$
\Phi: L^{q}(a, b) \rightarrow L^{q}(a, b ; w) \quad(1 \leq q<\infty, w \in \mathscr{W}(a, b))
$$

given by $\Phi(u)=u w^{-1 / q}$ is an isometric isomorphism. Using this map and Corollaries V.5.2, V.5.3 from [3] we see that the following analogue of Corollary V.5.4 of [3] is true.

Lemma 2.1. Let $P \in \mathscr{B}(X, Y)$, where $Y=L^{q}(a, b ; w), 1 \leq q<\infty, w \in$ $\mathscr{W}(a, b)$. Then $\operatorname{dist}(P, \mathscr{K}(X, Y))=\operatorname{dist}\left(P, \mathscr{F}_{r}(X, Y)\right)$.

Similarly, one can verify that the following analogue of Lemma V.5.6 from [3] holds.

Lemma 2.2. Set $Y=L^{q}(a, b ; w)$, where $1 \leq q<\infty, w \in \mathscr{W}(a, b)$. Let $P \in \mathscr{F}_{r}(X, Y)$ and $\epsilon>0$. Then there exist $R \in \mathscr{F}_{r}(X, Y)$ and $[\bar{a}, \bar{b}] \subset(a, b)$ such that $\|P-R\|<\epsilon$ and $\operatorname{supp} R f \subset[\bar{a}, \bar{b}]$ for all $f \in X$.

Further, we shall need

Proposition 2.3 (see [1, Theorem 2.21]). Let $1 \leq q<\infty$. A bounded set $S \subset$ $L^{q}(a, b)$ is precompact in $L^{q}(a, b)$ if, and only if, for every $\epsilon>0$ there exist a number $\delta>0$ and an interval $G=[\bar{a}, \bar{b}] \subset(a, b)$ such that for every $u \in S$ and every $h \in \mathbb{R}$ with $|h|<\delta$ we have

$$
\int_{a}^{b}|u(x+h)-u(x)|^{q} d x \leq \epsilon^{q}
$$

and

$$
\int_{(a, b) \backslash G}|u(x)|^{q} d x \leq \epsilon^{q} .
$$

( We define $u(x)=0$ for $x \notin(a, b)$ ).

\section{RESUltS}

First, let us formulate the main result of the paper.

Theorem 3.1. Suppose that $1<p \leq q<\infty,-\infty \leq a<b \leq \infty, v, w \in$ $\mathscr{W}(a, b), X=L^{p}(a, b ; v), Y=L^{q}(a, b ; w)$. Let $T$ be the operator (1.2) 
with the kernel $\varphi$ satisfying (1.3)(i)-(1.3)(iv). Moreover, let $B_{(a, b)}<\infty$. Then

$$
\frac{1}{2} J \leq \operatorname{dist}(T, \mathscr{K}(X, Y)) \leq k(q, p, D) J,
$$

where

$$
J=\lim _{\alpha \rightarrow a_{+}} B_{(a, \alpha)}+\lim _{\beta \rightarrow b_{-}} B_{(\beta, b)}
$$

and $k(q, p, D)$ is given by (1.8).

Corollary 3.2. Let all assumptions of Theorem 3.1 be fulfilled. Then

$$
T \in \mathscr{K}(X, Y) \Longleftrightarrow J=0 .
$$

Theorem 3.1 is a consequence of Lemmas 3.4 and 3.5.

From this point on we shall assume that symbols $a, b, p, q, T, X$, and $Y$ have the same meaning as in Theorem 3.1. Further, we shall need a certain decomposition of the operator $T$.

For any $\alpha, \beta \in(a, b), \alpha<\beta$, the operator $T$ can be written as

$$
T=T_{1}+T_{2}+T_{3} \text {, }
$$

where

$$
\begin{aligned}
& T_{1} f(x)=\chi_{(a, \alpha]}(x) \int_{a}^{x} \varphi(x, y) f(y) \chi_{(a, \alpha]}(y) d y, \\
& T_{2} f(x)=\chi_{(\alpha, b)}(x) \int_{a}^{x} \varphi(x, y) f(y) \chi_{(a, \beta]}(y) d y, \\
& T_{3} f(x)=\chi_{[\beta, b)}(x) \int_{a}^{x} \varphi(x, y) f(y) \chi_{[\beta, b)}(y) d y
\end{aligned}
$$

for $x \in(a, b)$; here $\chi_{M}$ is the characteristic function of the set $M$.

We have

Lemma 3.3. Suppose that the function $\varphi$ satisfies (1.3)(i)-(1.3)(iv). Moreover, let $B_{(a, b)}<\infty$. Then $T_{2} \in \mathscr{K}(X, Y)$.

Proof. The proof can be done by means of Proposition 2.3 (cf. the proof of Theorem 7.3 in [7] or the proof of Theorem 3 of [9]). Let us point out that assumption (1.3)(iv) is essential for this proof.

Now it is easy to establish an upper bound on $\operatorname{dist}(T, \mathscr{K}(X, Y))$.

Lemma 3.4. Suppose that the function $\varphi$ satisfies (1.3)(i)-(1.3)(iv). Let $B_{(a, b)}<$ $\infty$. Then

$$
\operatorname{dist}(T, \mathscr{K}(X, Y)) \leq k(q, p, D) J .
$$

Proof. Let $T_{2}$ be the operator from (3.5). According to Lemma 3.3, $T_{2} \in$ $\mathscr{K}(X, Y)$. Thus, using (3.3), we have

$$
\begin{aligned}
\operatorname{dist}(T, \mathscr{K}(X, Y)) & =\inf \{\|T-P\|: P \in \mathscr{K}(X, Y)\} \\
& \leq\left\|T_{1}+T_{3}\right\| \leq\left\|T_{1}\right\|+\left\|T_{3}\right\|
\end{aligned}
$$

with $T_{1}, T_{3}$ from (3.4), (3.6). However, each of the operators $T_{1}, T_{3}$ defines a bounded operator of the type given by (1.2). Therefore, by Theorem 1.1,

$$
\left\|T_{1}\right\| \leq k(q, p, D) B_{(a, \alpha)}, \quad\left\|T_{3}\right\| \leq k(q, p, D) B_{(\beta, b)} .
$$


Consequently, for any $\alpha, \beta \in(a, b)$ with $\alpha<\beta$,

$$
\operatorname{dist}(T, \mathscr{K}(X, Y)) \leq k(q, p, D)\left[B_{(a, \alpha)}+B_{(\beta, b)}\right],
$$

and (3.7) follows.

The following lemma provides a lower bound on $\operatorname{dist}(T, \mathscr{K}(X, Y))$.

Lemma 3.5. Assume that the function $\varphi$ satisfies (1.3)(i)-(1.3)(iii). Moreover, let $B_{(a, b)}<\infty$. Then

$$
\frac{1}{2} J \leq \operatorname{dist}(T, \mathscr{K}(X, Y))
$$

Proof. Let $\lambda>\operatorname{dist}(T, \mathscr{K}(X, Y))$. By Lemma 2.1 there exist $P \in \mathscr{F}_{r}(X, Y)$ such that

$$
\|T-P\|<\lambda \text {. }
$$

Set $\epsilon=(\lambda-\|T-P\|) / 2$. Then, according to Lemma 2.2, there exist $R \epsilon$ $\mathscr{F}_{r}(X, Y)$ and $[\bar{a}, \bar{b}] \subset(a, b)$ such that

$$
\|P-R\|<\epsilon
$$

and

$$
\operatorname{supp} R f \subset[\bar{a}, \bar{b}] \text { for every } f \in X \text {. }
$$

Inequalities (3.9) and (3.10) yield $\|T-R\|<\lambda$, and consequently,

$$
\|T f-R f\|_{Y} \leq \lambda\|f\|_{X} \text { for all } f \in X \text {. }
$$

This together with (3.11) implies that for all $f \in X$

$$
\int_{a}^{a}|T f(x)|^{q} w(x) d x+\int_{b}^{b}|T f(x)|^{q} w(x) d x \leq \lambda^{q}\|f\|_{X}^{q} .
$$

I. Take $\beta \in[\bar{b}, b), r \in(\beta, b), f \in X, f \geq 0$, and $i \in\{0,1\}$. Then

$$
\begin{aligned}
\int_{r}^{b}|T f(x)|^{q} w(x) d x & =\int_{r}^{b}\left(\int_{a}^{x} \varphi(x, y) f(y) d y\right)^{q} w(x) d x \\
& \geq \int_{r}^{b}\left(\int_{\beta}^{r} \varphi(x, y) f(y) d y\right)^{q} w(x) d x
\end{aligned}
$$

By (1.3)(ii) we have

$$
\varphi(x, y) \geq \varphi(r, y)^{1-i} \varphi(x, r)^{i}
$$

for $x>r>y$ and hence

$$
\int_{r}^{b}|T f(x)|^{q} w(x) d x \geq\left(\int_{r}^{b} \varphi(x, r)^{q i} w(x) d x\right)\left(\int_{\beta}^{r} \varphi(r, y)^{1-i} f(y) d y\right)^{q} .
$$

Now set

where

$$
f(y)=\varphi(r, y)^{\mu} v(y)^{1-p^{\prime}} \chi_{(\beta, r)}(y), \quad y \in(a, b),
$$

$$
\mu=(1-i) /(p-1)
$$

Then

$$
\|f\|_{X}^{q}=\left(\int_{\beta}^{r} \varphi(r, y)^{p^{\prime}(1-i)} v(y)^{1-p^{\prime}} d y\right)^{q / p}
$$


and, by (3.13),

$$
\begin{aligned}
\int_{r}^{b} \mid & \left.T f(x)\right|^{q} w(x) d x \\
& \geq\left(\int_{r}^{b} \varphi(x, r)^{q i} w(x) d x\right)\left(\int_{\beta}^{r} \varphi(r, y)^{p^{\prime}(1-i)} v(y)^{1-p^{\prime}} d y\right)^{q} .
\end{aligned}
$$

Thus from (3.12), (3.15), and (3.16) we obtain

$$
\lambda \geq\left(\int_{r}^{b} \varphi(x, r)^{q i} w(x) d x\right)^{1 / q}\left(\int_{\beta}^{r} \varphi(r, y)^{p^{\prime}(1-i)} v(y)^{1-p^{\prime}} d y\right)^{1 / p^{\prime}}=B_{(\beta, b)}^{(i)}(r)
$$

for any $r \in(\beta, b)$, for all $\beta \in[\bar{b}, b)$, and for $i \in\{0,1\}$. This shows that $B_{(\beta, b)} \leq \lambda$ for all $\beta \in[\bar{b}, b)$. Since $\lambda$ may be chosen arbitrarily close to $\operatorname{dist}(T, \mathscr{K}(X, Y))$, we obtain that $B_{(\beta, b)} \leq \operatorname{dist}(T, \mathscr{K}(X, Y))$ for every $\beta \in$ $[\bar{b}, b)$, and consequently,

$$
\lim _{\beta \rightarrow b_{-}} B_{(\beta, b)} \leq \operatorname{dist}(T, \mathscr{K}(X, Y)) .
$$

II. Take $\alpha \in(a, \bar{a}), r \in(a, \alpha), f \in X, f \geq 0$, and $i \in\{0,1\}$. Then, using (1.3)(ii), we obtain (cf. (3.13))

$$
\int_{r}^{\alpha}|T f(x)|^{q} w(x) d x \geq\left(\int_{r}^{\alpha} \varphi(x, r)^{q i} w(x) d x\right)\left(\int_{a}^{r} \varphi(r, y)^{1-i} f(y) d y\right)^{q}
$$

and, taking

$$
f(y)=\varphi(r, y)^{\mu} v(y)^{1-p^{\prime}} \chi_{(a, r)}(y), \quad y \in(a, b),
$$

with $\mu$ from (3.14), we get

moreover,

$$
\begin{aligned}
& \int_{r}^{\alpha}|T f(x)|^{q} w(x) d x \\
& \quad \geq\left(\int_{r}^{\alpha} \varphi(x, r)^{q i} w(x) d x\right)\left(\int_{a}^{r} \varphi(r, y)^{p^{\prime}(1-i)} v(y)^{1-p^{\prime}} d y\right)^{q}
\end{aligned}
$$

$$
\|f\|_{X}^{q}=\left(\int_{a}^{r} \varphi(r, y)^{p^{\prime}(1-i)} v(y)^{1-p^{\prime}} d y\right)^{q / p}
$$

Now, using (3.12), (3.18) and (3.19), we obtain (similarly as in part I)

$$
\lim _{\alpha \rightarrow a_{+}} B_{(a, \alpha)} \leq \operatorname{dist}(T, \mathscr{K}(X, Y)) \text {. }
$$

Together with (3.17) this yields (3.8), and the proof is complete.

Remark 3.6. If we suppose that

$$
\lim _{r \rightarrow a_{+}} B_{(a, b)}^{(i)}(r)=0=\lim _{r \rightarrow b_{-}} B_{(a, b)}^{(i)}(r)
$$

then it is easy to see that $J=0$. Thus, the assumptions of Theorem 3.1 and (3.20) imply that $T \in \mathscr{K}(X, Y)$.

On the other hand, if, for example, the function $\varphi$ satisfying (1.3)(i)(1.3)(iii) is bounded, then one can prove the implication $J=0 \Longrightarrow(3.20)$ (cf. the proof of Corollary (ii) from [5]).

Moreover, let us note that, using the arguments from [9], it is possible to prove the implication $T \in \mathscr{K}(X, Y) \Longrightarrow$ (3.20) provided the function $\varphi$ satisfies (1.3)(i)-(1.3)(iii).

Remark 3.6 and Corollary 3.2 imply 
Corollary 3.7. Let all assumptions of Theorem 3.1 be fulfilled. Then $T \in$ $\mathscr{K}(X, Y)$ if, and only if, (3.20) is true.

The statement of Corollary 3.7 was proved by V. D. Stepanov provided $\varphi(x, y)=k(x-y)$ with a suitable function $k$ (cf. [8]).

\section{REFERENCES}

1. R. A. Adams, Sobolev spaces, Academic Press, New York, San Francisco, and London, 1975.

2. S. Bloom and R. Kerman, Weighted norm inequalities for operators of Hardy type, Proc. Amer. Math. Soc. 113 (1991 135-141).

3. D. E. Edmunds and W. D. Evans, Spectral theory and differential operators, Oxford Univ. Press, Oxford, 1987.

4. D. E. Edmunds, W. D. Evans, and D. J. Harris, Approximation numbers of certain Volterra integral operators, J. London Math. Soc. (2) 37 (1988), 471-489.

5. R. K. Juberg, The measure of non-compactness in $L^{p}$ for a class of integral operators, Indiana Univ. Math. J. 23 (1974), 925-936.

6. F. J. Martin-Reyes and E. Sawyer, Weighted inequalities for Riemann-Liouville fractional integrals of order one and greater, Proc. Amer. Math. Soc. 106 (1989), 727-733.

7. B. Opic and A. Kufner, Hardy-type inequalities, Pitman Res. Notes in Math. Ser., vol. 219, Longman Sci. Tech., Harlow, 1990.

8. V. D. Stepanov, Weighted inequalities for a class of Volterra convolution operators, J. London Math. Soc. (2) 45 (1992), 232-242.

9. ___ Two-weighted estimates for Riemann-Liouville integrals, (Československá akademie věd) Matematický ústav, preprint no. 39, Praha, 1988, pp. 1-28.

10. C. A. Stuart, The measure of non-compactness of some linear integral operators, Proc. Roy. Soc. Edinburgh Sect. A 71 (1973), 167-179.

Mathematical Institute, Czech Academy of Sciences, Žrtná 25, 11567 Prague, Czech REPUBLIC

E-mail address: OPIC@CSEARN. BITNET 